\title{
Review
}

\section{First impressions on TOUSS approach of the oral cavity and pharyngeal malignant tumors}

\author{
Bogdan Popescu ${ }^{1,2}$, Irina Doinița Oașă ${ }^{1}$, Claudiu Curcă ${ }^{1}$, Elena M Arjoca ${ }^{1}$, Denisa M. \\ Mitran $^{1}$, Roxana G. Androne ${ }^{1}$, Șerban VG. Berteșteanu ${ }^{1,2}$, Răzvan V. Scăunașu ${ }^{2,3}$, \\ Alina LA. Oancea ${ }^{1,2}$ \\ ${ }^{1}$ Colţea Clinical Hospital, The ENT Department, Bucharest, Romania \\ ${ }^{2}$ Carol Davila University of Medicine and Pharmacy, Bucharest, Romania \\ ${ }^{3}$ Colţea Clinical Hospital, Department of General Surgery, Bucharest, Romania
}

\begin{abstract}
Oral and pharyngeal cancer are currently on an ascending slope in terms of incidence, mainly due to alcohol and tobacco consumption. In terms of risk factors, certain subtypes of HPV have been associated with an increased incidence of oral cancer.

Despite the knowledge of the methods of oral cancer prevention, screening is less efficient in some areas where medical services are unavailable or patients are not educated on the topic. This trans-oral robotic derived approach was introduced by M.M. Fernandez in 2014 as an alternative to the classical approach of the malignant tumors of the oral cavity, oropharynx and hypopharynx. In some selected cases, partial laryngectomy is an indication for using this technique.

The aim of this paper is to present some early considerations on this technique with indications, complications, advantages and disadvantages.

Keywords

: TOUSS, oral cancer, pharyngeal cancer, ultrasonic resection

Highlights

TOUSS is a prominent alternative to classical surgery, LASER surgery and TORS for head and neck cancer with great potential to bridge the open approach with LASER surgery.

$\checkmark$ The oncological outcome of this surgical procedure has to be determined in larger cohort studies.
\end{abstract}

To cite this article: Popescu B, Oașă ID, Curcă C, Arjoca EM, Mitran DM, Androne RG, Berteșteanu ȘVG, Scăunașu RV, Oancea LA. First impressions on TOUSS approach of the oral cavity and pharyngeal malignant tumors. J Clin Invest Surg. 2019; 4(2): 72-76. DOI: $10.25083 / 2559.5555 / 4.2 / 72.76$ 


\section{Introduction}

TOUSS (Trans-Oral Ultra-Sonic Surgery) has been presented as an alternative to the much more expensive TORS and to the available classical or LASER techniques. This is an endoscopic invasive technique aimed at treating both benign and malignant tumors in the oral cavity, pharynx and supraglottic larynx. It involves the use of a particularly designed retractor mounted in the oral cavity, a series of autostatic blades to ensure the easy access to different regions in the upper aero-digestive tract, an optical enhancer to obtain images from the approached cavities, forceps derived from laparoscopic surgery and the aid of a cutting and sealing device. The cutting device is the ThunderbeatTM scalpel which is successfully used in general surgery as a combined energy high precision cutting instrument. A multimodal approach of the malignant tumors of the head and neck region is the current standard and an individualized therapy plan is to be managed for each case of cancer. The improvement of the radiation techniques in recent years and the possibility of concurrent chemotherapy along with the preservation of the function have led to an increased use of radiochemotherapeutic approach of oropharyngeal cancer $(1,2)$. However, there is still a debate on the right therapeutic choice regarding radio-chemotherapy as a primary treatment or combined surgery and adjuvant radiotherapy with or without chemotherapy (3). A more modern approach of the malignancy of the upper aero-digestive tract is to ensure its function as much as possible without sacrificing the oncological result. For this consideration, transoral robotic surgery (TORS) has been used to safely remove malignant tumors with as little impairment as possible on breathing, phonatory and deglutition functions (4-6). While TORS is somewhat expensive and reserved for excellency centers of head and neck oncology, other techniques have been developed to surpass some disadvantages of TORS, mainly the cost and costeffectiveness considerations, which are not well described (7). This is where TOUSS comes into discussion. This transoral robotic derived approach was introduced by M.M. Fernandez in 2014 as an alternative to the classical approach of malignant tumors of the oral cavity, oropharynx and hypopharynx. In selected cases, partial laryngectomy is an indication for using this technique.

The aim of this paper is to present the early considerations on this technique with indications, complications, advantages and disadvantages. The technique setup was performed as described by M.M. Fernandez's study in 2014, using a pharyngo-laryngeal retractor (GyrusTM FK-retractor), Thunderbeat ${ }^{\circledR} 5 \mathrm{~mm} 35$ $\mathrm{cm}$, Olympus rigid laparoscopes with 0 and 30-degree vision and autostatic forceps for laparotomy. The basis of this prospective study was discussed with the Ethic Committee and the patients were advised on the procedure. Patients had already been diagnosed with malignant tumors of the oral cavity, oropharynx and hypopharynx and therapy planning was discussed by the Oncology Committee. The alternatives to TOUSS were presented to patients who were to undergo this particular therapy and an informed consent was signed by the patients. The inclusion criteria referred to the adult age, over 18 years of age, no prior radiotherapy, squamous cell carcinoma of the oral cavity and pharynx, favorable decision of the Oncology Committee for surgery. The invasion of the prevertebral fascia, the invasion of the common or internal carotid arteries, distant site metastasis, morbidities incompatible with general anesthesia, refusal of surgery by the patients were considered exclusion criteria.

\section{Discussions}

Significant TOUSS is a TORS derived method for the surgical removal of the benign and malignant tumors of the upper respiratory tract, depending on the extension of the tumor and less on its location. The tumors of the oral cavity, oropharynx, hypopharynx and supraglottic larynx have a proper approach of TOUSS in selected cases and with trained teams. The pharyngo-laryngeal retractor is the same one as that used with TORS and it ensures a clear view of the sub-sites of the aforementioned sites. A variety of blades mounted on the caudal part of the retractor and the correct location and dimensions of the tumor need to be known prior to surgery in order to correctly chose a particular blade. Therefore, practice with mounting the retractor needs to be done. In inexperienced hands, the fixation of the retractor can lead to teeth avulsion, the injury of lips and free margin of the tongue, the injury to the tumor with consecutive bleeding which burdens further maneuvers and might delay surgery. This is why the preoperative endoscopic examination of the upper aerodigestive tract needs to be performed, so that the precise location and volume of the tumor be assessed. CT or MRI scans are not better in evaluating the parameters needed for retractor mounting.

TOUSS was used by our team to reduce scarring in the regions of the face and neck by performing closed surgery, to prevent tracheostomy, to improve the healing process, to have a precise resection due to the direct view by the optical acquisition of images and digital subtraction (when using narrow band imaging filters). Since one of the aims of TOUSS is not to perform tracheostomy, retractor 
mounting has to take into consideration the positioning of the intubation tube. In our study, patients had oro-tracheal intubation with mechanical ventilation. We are considering the use of naso-tracheal intubation or jet ventilation for upper aero-digestive tract cancer patients in order to have an improved access to mount the pharyngo-laryngeal retractor and a better access in case of large tumors or bilateral involvement. A well-placed clip ensures the possibility of attaching a suction device for secretions and smoke resulted during surgery.

The endoscopic view is one of the key points in TOUSS surgery and it is available in a variety of setups. Our team used Olympus rigid $5 \mathrm{~mm}$ 2D laparoscopes with 0 and 30degree view angles without a flexible tip. Other laparoscopes available for the procedure are OlympusTM Endoeye Flex $5 \mathrm{~mm} / 2 \mathrm{D} 10 \mathrm{~mm} / 3 \mathrm{D}$ which have the advantage of a wider intraoperative surgical field perspective since the flexible tip of the laparoscope has a range of movement of 100 degrees. This was one of the problems in our rigid laparoscope setup, that is depending on the location and size of the tumor we needed to replace the laparoscope in the scope-holder during surgery. This is not the best way to perform surgery, since it is time consuming and, in some cases, we were unable to appreciate the correct extension of the tumor. Still, after the repositioning of the laparoscope, we were able to obtain optimal images to perform surgery (Figure 1).

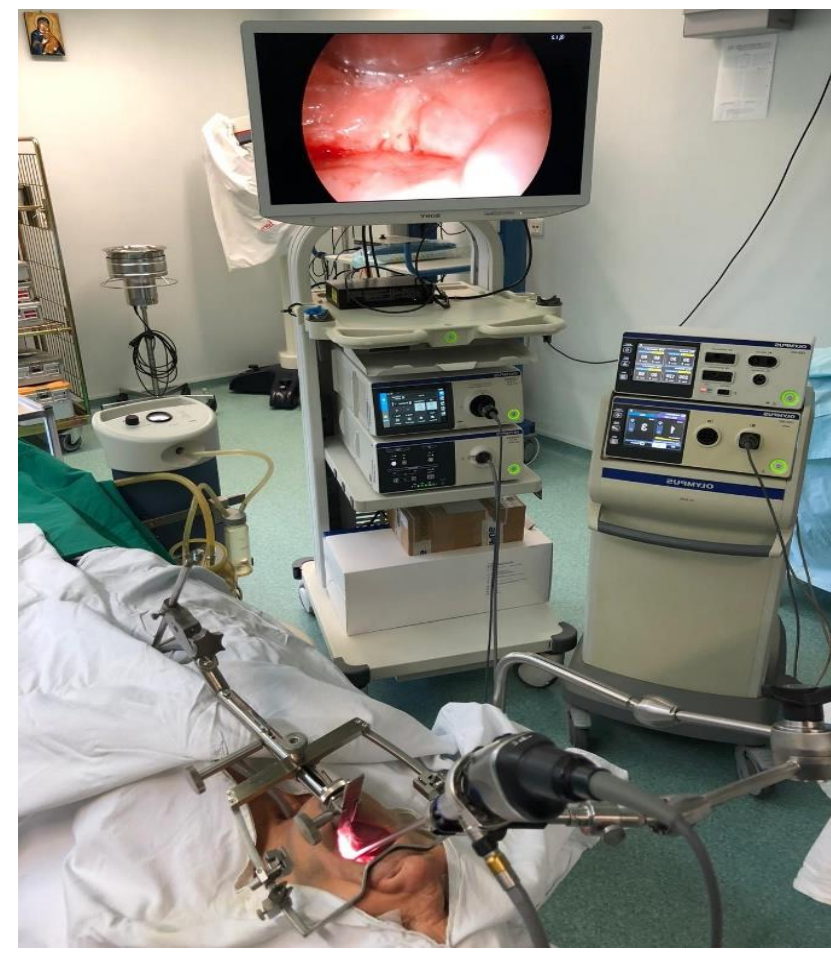

Figure 1. TOUSS setup with pharyngo-laryngeal retractor and 0-degree rigid laparoscope mounted. Endoscopic view with $4 \mathrm{k}$ resolution and $2 \mathrm{D}$ vision.
The instruments used to resect the tumor are derived from laparoscopic fixed arm in the shape of a needle, which generates cutting capabilities. The cutting of the tissue is a result of vibrating mechanical energy generated at high 25$55 \mathrm{kHz}$ frequency with the advantage of lower temperature generation and a smaller thermal injury of the surrounding tissue. The maximal temperatures of Thunderbeat TM scalpel are about 200 degrees Celsius and the lateral spread of the heat is up to $2 \mathrm{~mm}$. The thermal properties of Thunderbeat TM scalpel allow surgeons to resect tumors close to large blood vessels and even if there is the need to resect these vessels, the capabilities of the device mean that arteries up to $7 \mathrm{~mm}$ can be cut and sealed safely. Surgeons can adjust the parameters of cutting and coagulation individually, therefore optimized resection can be performed. A lateral spread of the thermal energy in the 2 $\mathrm{mm}$ range means that even tumors located closest to the carotid artery can be resected with some degree of safety. Along with the ultrasonic resection capabilities, Thunderbeat TM scalpel incorporates an advanced bipolar sealing system that can be activated simultaneously or independently (8).

\section{The surgical procedure}

In terms of the setup required to begin surgery, TOUSS has been reported to be as low as 5 minutes (9) in comparison to the median time of 10-minute setup for TORS (10). However, this time for setup has been achieved by surgical teams specially trained in the procedure. In our operating room, the average time for setup considering 6 cases was 11.2 minutes. In 4 cases out of the 6 operated in our clinic, the resection referred to as the carcinoma of the oral cavity, oropharynx and hypopharynx, with 2 cases of epiglotectomy, 1 for the upper airway obstruction following radiotherapy for supraglottic cancer of the larynx and 1 case for Kaposi sarcoma. In all of the 4 cases operated on for squamous cell carcinoma, the tumor resection followed neck dissection for local lymph node metastasis confirmed both clinically and by means of CT scans. No tracheostomy was performed. TOUSS setup was the same in all cases in regard to the access to the operating site and step one or step two of surgery. Still, in both cases of epiglotectomy, the TOUSS system setup was easier since the location of the lower blade of the retractor was in the midline and there was no bulky or bleeding tumor.

The endoscopic view was sufficient in 4 cases and did not need rearrangement of the laparoscope. This may be linked to the fact that we used 0 and 30 degree rigid laparoscopes or to the size of the tumor. The larger angle view optical devices such as the Olympus ENDOEYE Flex 
$5 \mathrm{~mm}$ 2D or ENDOEYE Flex $10 \mathrm{~mm}$ 3D video laparoscopes are yet to be used in a common setup by our surgical team. One of the key aspects of the technique is that surgical planes and the relation between the organs and the surrounding tissue needs to be precisely assessed, since the depth of dissection is mandatory to obtain a clear resection margin and the jugular-carotid space is in close relation to the lateral wall of the pharynx. This is why a $3 \mathrm{D}$ setup is more precise for a more accurate dissection, while the depth of the field is essential in appreciating the relation with the large vessels of the neck. TOUSS setup implies the need of using forceps to grasp and hold the tissue. These forceps are the same as those for abdominal laparoscopic surgery. They are adequately long and allow the rotation of the tip of the forceps in the long axes of the forceps which implies a 360-degree freedom of movement in the optical field. However, in inexperienced hands, a longer time to orient and coordinate the surgical instruments in the operating field is necessary, thus a longer overall time of surgery is required. At this moment, we are unable to establish a reasonable time for a particular type of surgery. Further analysis needs to be made since there is no standard time for the procedure (Figure 2).

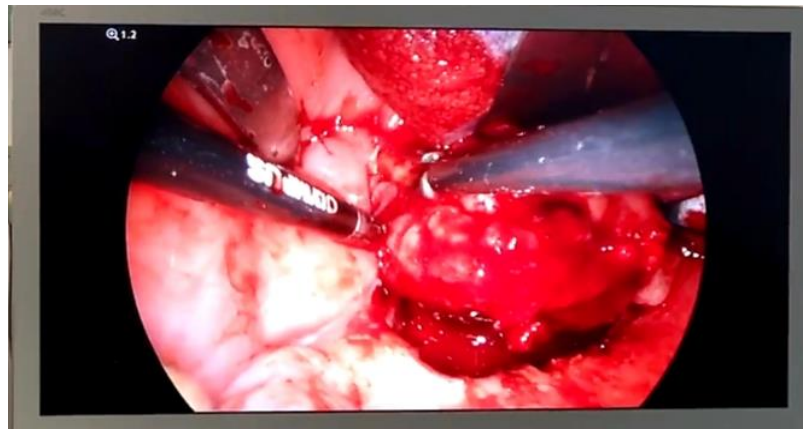

Figure 2. Squamous cell carcinoma of the tongue and left lateral wall of the oropharynx and hypopharynx ( $4 \mathrm{k}$ resolution with 1.2 magnification in $2 \mathrm{D}$ view)

In terms of bleeding, we replaced the classical external carotid ligation approach for cutting blood supply to the tumor with the cutting and sealing capabilities of Thunderbeat TM. The primary site or the extension of the tumor at the level of the tongue usually resides in a bloody resection. There was no additional bleeding after resecting the tumors with the ultrasonic scalpel.

\section{OUSS follow-up}

Oral cavity and pharyngeal cancer patients operated on with TOUSS had a similar recovery time in terms of the hospitalization period, the use of the naso-gastric tube and functional recovery. This is because we decided to follow up patients the same way we monitor patients with similar pathology operated on through a classical approach. The data are to be confirmed by further studies and the efficacy profiles are to be analyzed.

\section{Conclusions}

Since this type of approach of benign and malignant tumors of the oral cavity, oropharynx and hypopharynx is yet to be standardized, a larger number of procedures for particular sites and types of tumors need to be performed. Preliminary assessments suggest that TOUSS is a feasible and effective technique that may compete TORS in terms of efficacy and the oncological outcome. The main benefit of TOUSS in comparison with TORS is the purchase price, even with economic profile debated for the costeffectiveness of TORS (7).

The learning curve of TOUSS is not steep, but it needs to be addressed in excellency centers of oncology through specific procedures performed by well-trained teams. The ability of having a digital enhanced operating field accompanied by the performance of ultrasonic scalpel makes us believe that there is a great possibility for a new, oncological, effective, safe procedure that decreases the hospitalization period and morbidity rates and increases the quality of the life of patients. There is still the need to evaluate the overall survival rates for cancer patients who undergo TOUSS. Another upside of the procedure is that we were able to assess the blood vessels surrounding the tumor in the mucosa, prior to the first incision and to appreciate the potential local spread of the malignant process. This was achieved by using narrow band imaging (NBI) along with the regular endoscopic view. We are yet to assess the safety margins for each organ while using NBI.

TOUSS is a prominent alternative to classical surgery, LASER surgery and TORS for head and neck cancer with great potential to bridge the open approach with LASER surgery. The oncological outcome of the procedure has to be determined in larger cohort studies.

\section{Conflict of interest disclosure}

There are no known conflicts of interest in the publication of this article. The manuscript was read and approved by all authors.

\section{Compliance with ethical standards}

Any aspect of the work covered in this manuscript has been conducted with the ethical approval of all relevant bodies and that such approvals are acknowledged within the manuscript. 


\section{References}

1. Forastiere AA, Trotti A. Radiotherapy and concurrent chemotherapy: a strategy that improves locoregional control and survival in oropharyngeal cancer. J Natl Cancer Inst. 1999; 91(24): 2065-2066.

2. Parsons JT, Mendenhall WM, Stringer SP, et al. Squamous cell carcinoma of the oropharynx: surgery, radiation therapy, or both. Cancer. 2002; 94(11): $2967-$ 2980.

3. Koch WM. Head and neck surgery in the era of organ preservation therapy. Semin Oncol. 2000; 27(4 Suppl 8): 5-12.

4. Genden EM, Shaun Desai BA, et al. Transoral robotic surgery for the management of head and neck cancer: preliminary experience. Head Neck. 2009; 31(3): 283 289.

5. Weinstein GS, O'Malley BW, Cohen MA, et al. Transoral robotic surgery for advanced oropharyngeal carcinoma. Arch Otolaryngol Head Neck Surg. 2010; 136(11): 1079-1085.

6. Dowthwaite SA, Franklin JH, Palma DA, et al. The role of transoral robotic surgery in the management of oropharyngeal cancer: a review of the literature.
ISRN Oncol. 2012; 2012: 945162-945162. DOI: $10.5402 / 2012 / 945162$

7. Byrd JK, Smith KJ, de Almeida JR, Albergotti WG, Davis KS, Kim SW et al. Transoral robotic surgery and the unknown primary: a cost-effectiveness analysis. Otolaryngol Head Neck Surg. 2014; 150(6): 976-982.

8. Seehofer D, Mogl M, Boas-Knoop S, Unger J, Schirmeier A, Chopra S et al. Safety and efficacy of new integrated bipolar and ultrasonic scissors compared to conventional laparoscopic 5-mm sealing and cutting instrument. Surg Endosc. 2012; 26(9): 2541-2549.

9. Mario M. Fernandez-Fernandez, Lourdes MontesJovellar, Pablo Luis Parente Arias, Primitivo Ortega del Alamo. TransOral endoscopic UltraSonic Surgery (TOUSS): a preliminary report of a novel robotless alternative to TORS. Eur Arch Otorhinolaryngol. 2015; 272(12): 3785-3791.

10. More YI, Tsue TT, Girod DA, Harbison J, Sykes KJ, Williams $\mathrm{C}$ et al. Functional swallowing outcomes following transoral robotic surgery vs primary chemoradiotherapy in patients with advanced-stage oropharynx and supraglottis cancers. JAMA Otolaryngol Head Neck Surg. 2013; 139(1): 43-8. DOI: 10.1001/jamaoto.2013.1074 